\title{
Asymmetric Localization of Vangl2 and Fz3 Indicate Novel Mechanisms for Planar Cell Polarity in Mammals
}

\author{
Mireille Montcouquiol, ${ }^{1,2}$ Nathalie Sans, ${ }^{3}$ David Huss, ${ }^{4}$ Jacob Kach, ${ }^{5}$ J. David Dickman, ${ }^{4}$ Andrew Forge, ${ }^{6}$ \\ Rivka A. Rachel, ${ }^{7}$ Neal G. Copeland, ${ }^{7}$ Nancy A. Jenkins, ${ }^{7}$ Debora Bogani, ${ }^{8,9}$ Jennifer Murdoch, ${ }^{9}$ Mark E. Warchol, ${ }^{5}$ \\ Robert J. Wenthold, ${ }^{3}$ and Matthew W. Kelley ${ }^{1}$ \\ ${ }^{1}$ Section on Developmental Neuroscience, National Institute on Deafness and Other Communication Disorders, National Institutes of Health, Bethesda, \\ Maryland 20892, 2Equipe Avenir 2 "Neurosciences Développementales," Institut F. Magendie des Neurosciences, Institut National de la Santé et de la \\ Recherche Médicale, 33077 Bordeaux Cedex, France, ${ }^{3}$ Laboratory of Neurochemistry, National Institute on Deafness and Other Communication Disorders, \\ National Institutes of Health, Bethesda, Maryland 20892, Departments of ${ }^{4}$ Anatomy and Neurobiology and ${ }^{5}$ Otolaryngology, Washington University School \\ of Medicine, St. Louis, Missouri 63110, ${ }^{6}$ Centre for Auditory Research, UCL Ear Institute, University College London, London WC1X 8EE, United Kingdom, \\ ${ }^{7}$ Mouse Cancer Genetics Program, National Cancer Institute-Frederick, Frederick, Maryland 21702, and ${ }^{8}$ Early Development, Mammalian Genetics Unit, \\ ${ }^{9}$ Medical Research Council, Harwell, Oxfordshire OX11 0RD, United Kingdom
}

Planar cell polarity (PCP) is a process in which cells develop with uniform orientation within the plane of an epithelium. To begin to elucidate the mechanisms of PCP in vertebrates, the localization of the protein Vangl2 (Van Gogh-like) was determined during the development of the mammalian cochlea. Results indicate that Vangl2 becomes asymmetrically localized to specific cell-cell boundaries along the axis of polarization and that this asymmetry is lost in PCP mutants. In addition, PDZ2 (postsynaptic density/Discs large/zona occludens 1), PDZ3, and PDZ4 of the PCP protein Scrb1 (Scribble) are shown to bind to the C-terminal PDZ binding domain of Vangl2, suggesting that Scrb1 plays a direct role in asymmetric targeting of Vangl2. Finally, Fz3 (Frizzled), a newly demonstrated mediator of PCP, is also asymmetrically localized in a pattern that matches that of Vangl2. The presence and asymmetry of Fz3 at the membrane is shown to be dependent on Vang12. This result suggests a role for Vang12 in the targeting or anchoring of Fz3, a hypothesis strengthened by the existence of a physical interaction between the two proteins. Together, our data support the idea that protein asymmetry plays an important role in the development of PCP, but the colocalization and interaction of Fz3 and Vangl2 suggests that novel PCP mechanisms exist in vertebrates.

Key words: inner ear; stereociliary bundle; mechanosensory hair cell; cochlea; Van Gogh; Frizzled; planar polarity

\section{Introduction}

One of the clearest examples of planar cell polarity (PCP) in a vertebrate system is the sensory epithelium of the mammalian cochlea [also referred to as the organ of Corti (OC)]. The OC exists as a narrow strip of cells that extends along the coiled basalto-apical axis of the cochlear duct. The OC itself comprises multiple cell types, including inner and outer mechanosensory hair cells (IHCs and OHCs, respectively) and nonsensory supporting cells (SCs), including inner phalangeal cells (IPh), inner and

\footnotetext{
Received Nov. 1, 2005; revised March 22, 2006; accepted March 25, 2006.

This work was supported in part by the Intramural Research Program at the National Institute on Deafness and Other Communication Disorders (NIDCD) (M.W.K., M.M., R.J.W., N.S.), the Intramural Research Program at the National Cancer Institute, Center for Cancer Research (R.A.R., N.G.C., N.A.J.), the Defeating Deafness Foundation (A.F.), NIDCD Research grants (M.E.W., J.D.D.), the P30 Inner Ear Consortium Grant to Washington University (M.E.W., J.D.D.), and the Medical Research Council (J.M.). We thank Drs. Doug Cotanche, Doris Wu, and Ronna Hertzano for comments on this manuscript.

Correspondence should be addressed to Mireille Montcouquiol, Equipe Avenir 2 “Neurosciences Développementales," Institut F. Magendie des Neurosciences, Institut National de la Santé et de la Recherche Médicale, 33077 Bordeaux Cedex, France. E-mail: montcouquio@bordeaux.inserm.fr.

N. Sans's present address: Equipe Avenir 3 "Neurobiologie Cellulaire et Moléculaire," Institute F. Magendie des Neurosciences, Institut National de la Santé et de la Recherche Médicale, 33077 Bordeaux Cedex, France.

DOI:10.1523/JNEUROSCI.4680-05.2006

Copyright $\odot 2006$ Society for Neuroscience $\quad 0270-6474 / 06 / 265265-11 \$ 15.00 / 0$
}

outer pillar cells (IPs and OPs, respectively), and Deiters cells (see Fig. $1 \mathrm{~A}$ ). All cell types are arranged in rows that extend along the length of the cochlear spiral. Each HC contains a bundle of modified microvilli, referred to as stereocilia, that project from its lumenal surface. The individual stereocilia are arranged in a specific pattern such that each stereociliary bundle has a shape that is similar to a ")". For each hair cell, the vertex of the bundle is located on the distal edge (see Fig. 1A). The presence of these uniformly oriented bundles illustrates the high degree of PCP that exists within the $\mathrm{OC}$ and makes this system an ideal choice for the study of mechanisms that regulate PCP in vertebrates.

Although significant progress has been made toward understanding the molecular and biochemical bases for the development of PCP in invertebrate systems, similar advances in vertebrate systems have not occurred, primarily as a result of a lack of appropriate PCP mutants. Recent results have identified a group of mammalian PCP mutants that affect HC stereociliary bundle orientation, including Vangl2 (Van Gogh-like) Scrb1 (Scribble), Celsr 1 (cadherin EGF LAG seven-pass G-type receptor), and protein tyrosine kinase-7 (PTK-7) (Curtin et al., 2003; Montcouquiol et al., 2003; Lu et al., 2004). It has also been reported that there are orientation defects in Dvll/2 (Dishevelled) double mu- 
tants (Wang et al., 2005), but the overall level of disruption appears mild in these animals and may be complicated by functional redundancy and additional roles for Dvl. Whereas the PCP functions of Vangl2 and Celsr1 seem to have been maintained between vertebrates and invertebrates, $S c r b 1$ and PTK-7 are apparently novel vertebrate PCP genes that have no role in PCP in Drosophila (Montcouquiol et al., 2003; Lu et al., 2004). Together, these results and our data suggest that, although there has been an overall conservation of function in terms of the molecular pathways that generate PCP, significant differences probably exist between vertebrates and invertebrates. In this study, we sought to begin to determine the cellular interactions that mediate PCP in vertebrates through an analysis of the localization of Vangl2 during the generation of PCP in the mammalian cochlea.

\section{Materials and Methods}

Looptail and circletail animals. Looptail (Lp) mutant mice on the LPT/Le stock were obtained from The Jackson Laboratory (Bar Harbor, ME). The circletail mutation arose spontaneously in 1997 in a transgenic colony at Columbia University (New York, NY) (Rachel et al., 2002). Both strains of mice were maintained at the National Institutes of Health under standard conditions by heterozygous intercross. To generate homozygous mutants, heterozygous animals were crossed and timedpregnant litters were obtained at embryonic day 18.5 (E18.5). Heterozygous offspring were identified by a looped tail, whereas homozygous fetuses die perinatally and were distinguished embryonically by the presence of craniorachischisis (Strong and Hollander, 1949; Copp et al., 1994). Rats were obtained from Harlan (Indianapolis, IN).

Vangl2 and Scrb1 antibody characterization. Vangl2 and Scrb1 antibodies were generated by immunizing rabbits with the peptide corresponding to amino acids 411-423 and 1650-1665, respectively, and affinity purified on a column of covalently coupled peptide. Vangl2 antibody recognized the tagged and untagged protein when expressed in various cell lines, including Chinese hamster ovary (COS1), MadinDarby canine kidney (MDCK), human embryonic kidney (HEK293), and normal rat kidney (NRK) cell lines, as well as a band at $\sim 65 \mathrm{kDa}$ in brain and cochlear lysates. Scrb1 antibody recognized a band at $\sim 200$ $\mathrm{kDa}$ in brain and cochlear lysates, as well as the untagged protein when expressed in HEK293 cells.

Immunocytochemistry and immunohistochemistry. Cochleae from rats and mice were dissected from wild-type (WT) and mutant littermates at specific time points between E18.5 and postnatal 10 (P10) and were fixed in $2 \%$ paraformaldehyde in PBS for $4 \mathrm{~h}$ at $4^{\circ} \mathrm{C}$. Some cochleae were processed as whole mounts, whereas others were cryoprotected through a sucrose gradient, embedded in O.C.T. (Electron Microscopy Sciences, Hatfield, PA), and sectioned in a cryostat at a thickness of $14 \mu \mathrm{m}$.

Whole-mount immunostaining of entire cochleae was performed as described previously (Woods et al., 2004) using the following primary antibodies: anti-p75 ${ }^{\text {ntr }}$ (1:1000; Chemicon, Temecula, CA), anti-Ncadherin (1:500; Chemicon), anti-Vangl2 (1:500; this study), anti-Scrb1 (1:500, this study; 1:100, Santa Cruz Biotechnology, Santa Cruz, CA), anti- $\beta$-catenin (1:500; Chemicon), anti-Fz3 (Frizzled) (1:200; Sigma, St. Louis, MO), and anti- $\beta 1 / \beta 2$-tubulin (1:200; Sigma). Antibody binding was detected by using an Alexa Fluor-594- or Alexa Fluor-488conjugated secondary antibody (Invitrogen, Carlsbad, CA) at a dilution of $1: 1000$. When comparing staining between WT and mutants, both cochleae were processed in the same tube.

Cryostat sections were permeabilized with $0.5 \%$ Triton X-100 in PBS, blocked with $10 \%$ normal goat serum (NGS), and then incubated overnight at $4^{\circ} \mathrm{C}$ in primary antibodies. Antibody labeling was visualized as described. Alexa Fluor-488 or Alexa Fluor-594-conjugated phalloidin (1:1000; Invitrogen) was used to visualize cellular borders in cryosections. All sections were photographed on a Zeiss (Thornwood, NY) LSM510 confocal microscope. Digital images were processed in Adobe Photoshop (Adobe Systems, San Jose, CA).

Scanning electronic microscopy. Utricles were harvested from Van$g l^{L / L P}$ and Vangl2 $^{L P /+}$ mouse embryos at E18.5 and placed in cold PBS.
After removal of the otoconia and otolithic membranes, specimens were fixed in $2.5 \%$ glutaraldehyde, dehydrated through a graded acetone series, washed in tetramethylsilane, and allowed to sublimate at $37^{\circ} \mathrm{C}$. Dried utricles were then sputter coated with palladium and imaged on a Hitachi (Yokohama, Japan) 2600 scanning electron microscope at $15 \mathrm{kV}$. A series of contiguous scanning electron microscopic images were obtained from each specimen, along an axis that was parallel to the lateromedial axis and approximately midway between the anterior and posterior poles (see Fig. 2). These images were then joined together, resulting in a sampled region in each utricle that was linear, $\sim 200 \mu \mathrm{m}$ long and 30 $\mu \mathrm{m}$ wide, and perpendicular to the striolar reversal zone. The angular orientation of each stereocilia bundle within these regions was then quantified following the method of Denman-Johnson and Forge (1999). Data were obtained from three $\operatorname{Vangl}^{L p / L p}$ and three $\operatorname{Vangl}^{L p /+}$ utricles ( $n=32-101$ stereocilia bundles per specimen).

In situ hybridization. Embryos from pregnant CD-1 mice (Charles River Laboratories, Wilmington, MA) were staged individually, and cochleae were dissected and processed for in situ hybridization as a whole mount as described previously (Hertzano et al., 2004). The tissue was then cryosectioned at a thickness of $12 \mu \mathrm{m}$. A digoxigenin-labeled antisense RNA probe for Vangl2 was generated from a phosphorylated cytomegalovirus (CMV) SPORT6 vector carrying a $1 \mathrm{~kb}$ fragment of the coding region (expressed sequence tag BF118609).

Cell culture and transfections. HEK293, COS1, COS7, or MDCK cells were grown on $10 \mathrm{~cm}$ dishes for biochemical analyses or on glass coverslips in 24-well tissue culture dishes for immunofluorescence microscopy. Cells were transfected using the Lipofectamine 2000 (Invitrogen) according to the procedures of the manufacturer or the calcium phosphate coprecipitation method and were analyzed 24 or $48 \mathrm{~h}$ after transfection.

Immunofluorescence of MDCK cells. For intracellular labeling, transfected cells were fixed with $4 \%$ paraformaldehyde, washed, permeabilized in $0.5 \%$ Triton X-100/PBS, and blocked in 10\% NGS/PBS for $1 \mathrm{~h}$. The cells were then incubated with appropriate primary antibodies for $1 \mathrm{~h}$ in $2 \% \mathrm{NGS} / \mathrm{PBS}$, washed, and then incubated in secondary antibodies for 30 min (Alexa Fluor-488 and -594) in 2\% NGS/PBS.

cDNA constructs, culture, and electroporation. Briefly, E18.5 mouse cochlear epithelia were isolated with thermolysin treatment as described previously (Montcouquiol et al., 2003), and the mRNA was extracted and treated with reverse transcriptase to obtain a pool of cDNA. Full-length Vangl2 was amplified from this pool and cloned into the mammalian expression vectors pcDNA3.1, pGFP-C3 (Clontech, Cambridge, UK), and pCLIG. The serine S464 of Vangl2 was replaced by an asparagine to generate Vangl2-S464N (Vangl2 ${ }^{L P}$ ) using Quickchange II site-directed mutagenesis kit according to the protocol of the manufacturer (Stratagene, La Jolla, CA). The Scrb1 expression construct was generously provided by Dr. Cornelia Kurschner (St. Jude's Hospital, Memphis, TN), and the Fz3 expression construct was generously provided by Dr. Jeremy Nathans (Johns Hopkins University, Baltimore, MD).

Cochlear explant cultures were established from E13.5 and E14.5 embryos as described previously (Montcouquiol et al., 2003). Individual cells were transfected with Vangl 2 or Vangl2 ${ }^{L p}$ constructs by square-wave electroporation. Dissected explants were placed in a $10 \mu \mathrm{l}$ drop containing $2 \mu \mathrm{g} / \mu \mathrm{l}$ circular DNA. The electrodes were placed at the edges of the $10 \mu \mathrm{l}$ drop, and a series of nine pulses with a voltage of $28 \mathrm{mV}$ and duration of $28 \mathrm{~ms}$ per pulse was applied. Transfected cells were identified using an anti-green fluorescent protein (GFP) antibody conjugated to Alexa Fluor-488 (Invitrogen). Cell boundaries and stereociliary bundles were visualized with phalloidin conjugated to Alexa Fluor-488 or -594 (Invitrogen).

Two-hybrid experiment. Vangl2-Ct (amino acids 1580-1665) was subcloned into pGBTK7 in-frame with the DNA binding domain of galactosidase-4 (GAL4). Yeast two-hybrid screening and assays were performed as described in the Clontech protocol. AH109 cells expressing GAL4-Vang12 C-term were combined with Y187 cells expressing the mouse E17 embryo cDNA library. The mating mixture was plated on $\mathrm{SD} /-$ Ade/ $-\mathrm{Trp} /-\mathrm{Leu} /-$ His plates. From $7 \times 10^{6}$ colonies obtained $5 \mathrm{~d}$ after transformation, 148 colonies tested His positive/LacZ positive. The 
A
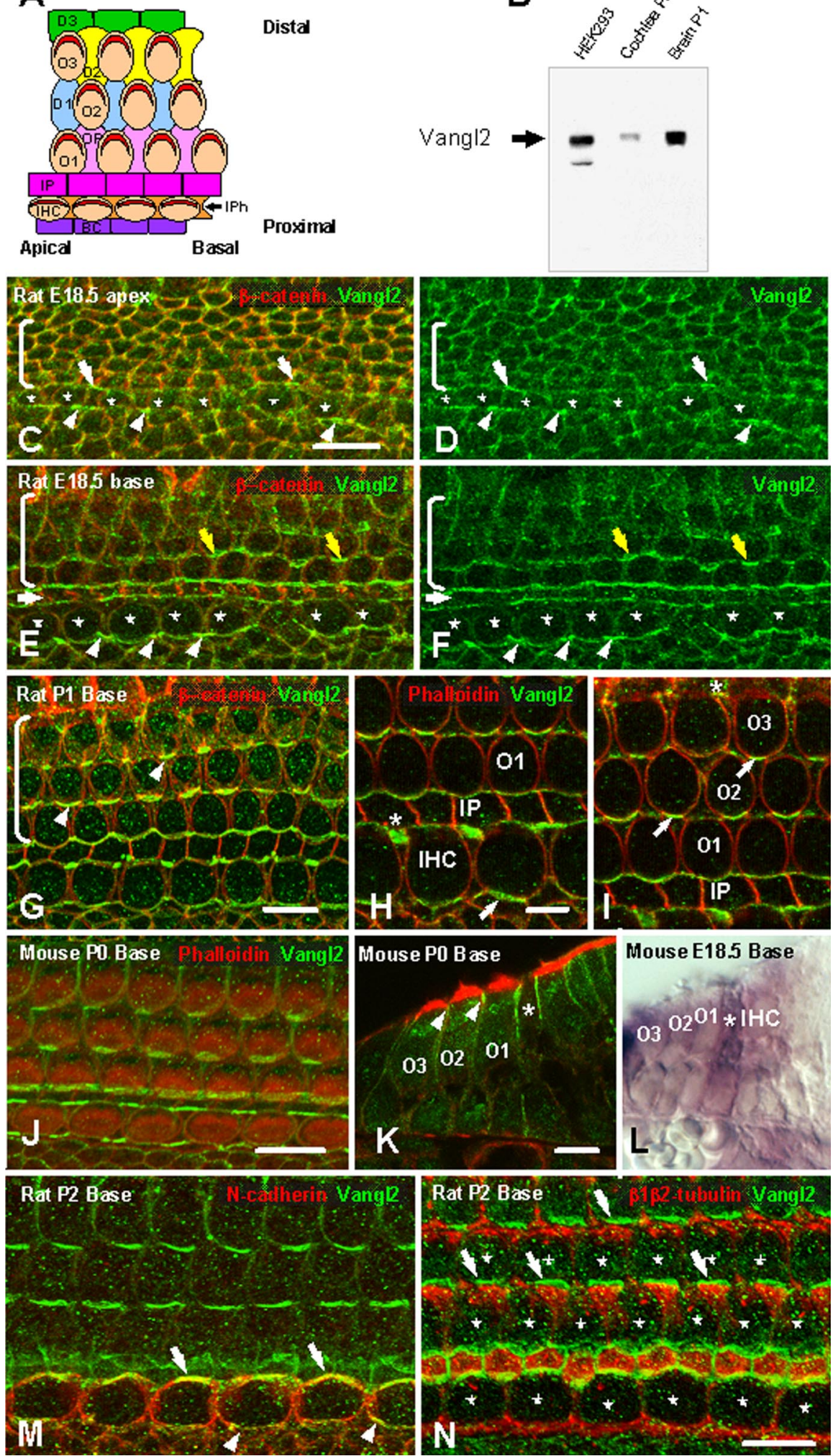

$0302 \quad 01$

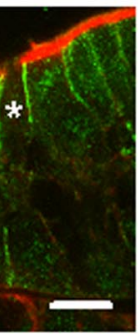

Mouse E18.5 Base
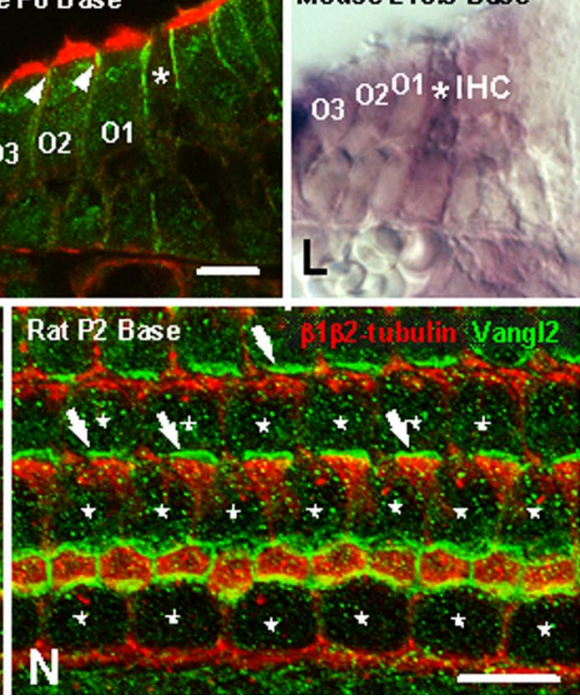

Figure 1. VangI2 is asymmetrical localized in the organ of Corti. $A$, Schematic diagram illustrating a surface view of the $O C$ in the mammalian cochlea. Proximal-to-distal and basal-to-apical axes are indicated. The $\mathrm{OC}$ comprises a single row of $\mathrm{IHC}$ and three rows of $\mathrm{OHCS} \mathrm{(01-03)} \mathrm{that} \mathrm{extend} \mathrm{along} \mathrm{the} \mathrm{basal-to-apical} \mathrm{axis.} \mathrm{Individual} \mathrm{hair} \mathrm{cells} \mathrm{are} \mathrm{separated} \mathrm{by} \mathrm{specific} \mathrm{types} \mathrm{of}$ supporting cells including IPh cells located between IHCS, Deiters cells (D1-D3), located between OHCs and OPs, located between $\mathrm{OHCs}$ in $\mathrm{OHC1}$ and IPs, which create a space between the row of IHC and $\mathrm{OHC1}$. Finally, the proximal edge of IHCs is contacted by a row of border cells (BC). Each $\mathrm{HC}$ has a stereociliary bundle (red) located on its lumenal surface. Each bundle is oriented such that its central vertex is located closest to the distal edge of the epithelium. $\boldsymbol{B}$, Affinity-purified Vangl2 antibody recognizes a band $\sim 65 \mathrm{kDa}$ in lysates from HEK293 transfected with an untagged Vangl2 construct (lane 1), cochlea (lane2), and brain (lane 3). C, $\boldsymbol{D}$, Lumenal surface of the $0 C$ in the apical turn of a rat cochlea at E18.5. Vangl2 (green) is colocalized with $\beta$-catenin (red) at the library plasmids from those colonies were rescued, amplified by PCR, and sequenced.

$\beta$-Galactosidase assay. $\beta$-Galactosidase activity was measured in cotransformed yeast colonies by using the Gal-Screen assay system (Tropix, Bedford, MA). Three 1-2 mm colonies were placed in $2 \mathrm{ml}$ of glucose - Trp/-Leu conditioned medium and incubated for $12-16 \mathrm{~h}$ at $30^{\circ} \mathrm{C}$ until $\mathrm{OD}_{600} 0.45-0.50$. Gal-Screen Buffer $\mathrm{B}$ was prepared according to the instructions of the manufacturer. The $100 \mu \mathrm{l} /$ well reaction Buffer B was combined with $100 \mu \mathrm{l} /$ well yeast culture and incubated for $90 \mathrm{~min}$ in solid-white 96-well microplates. Microplates were placed in a Tropix luminometer, and light emission kinetics were measured for $1 \mathrm{~s} /$ well. Singletransformed p53 and pBGKT7 colonies were used as positive and negative controls, respectively. All experiments were performed in triplicate.

Western blot and immunoprecipitation. Brain of P1 Sprague Dawley rats were homogenized in PBS containing a mixture of protease inhibitors (Sans et al., 2001), and protein concentrations were measured using a BCA assay (Pierce, Rockford, IL). For immunoprecipitation experiments, rat brains were processed using sodium deoxycholate solubilization as described previously (Sans et al., 2003). Immunoprecipi-

membranes of all epithelial cell types in the 0 C. Some asymmetric localization of VangI 2 is present in IPh cells (arrows) and IHCS (asterisks). In contrast, a uniform distribution of Vangl2 is present in the undifferentiated outer hair cell region (brackets). $\boldsymbol{E}, \boldsymbol{F}, \mathrm{In}$ the more mature basal turn of the same E18.5 rat cochlea, in which overall development is more advanced by comparison with the apical turn, the asymmetry of Vangl 2 is more pronounced in the IPh cells (arrowheads) but is also apparent on the proximal sides of the IHCs (asterisks), as well as in the pillar cells (white arrow) and the second row $\mathrm{OHC}$ (yellow arrows). $G$, At $P 1$, in the basal turn of the rat cochlea, there is an accumulation of Vangl2 at the junction between the proximal edges of the hair cells and distal edges of the supporting cells (arrowheads). Vangl2 protein is noticeably absent from the lateral sides of all cells as well as from the distal edges of hair cells and the proximal edges of supporting cells. $\boldsymbol{H}, \boldsymbol{I}$, Highmagnification view of the $\mathrm{IHC}$ region $(\boldsymbol{H})$ and $\mathrm{OHC}$ region $(\boldsymbol{I})$ in a $\mathrm{P} 1$ rat cochlea in single focal planes. $\beta$-Catenin labeling indicates the accumulation of Vangl2 at the exact junction between a supporting cell and a hair cell (arrows) but also between two supporting cells (asterisk). J, A similar asymmetric distribution of Vangl2 (green) is present in the basal turn of the mouse c0chlea at PO. $\boldsymbol{K}$, Cryostat section of a PO mouse cochlea showing the apical localization of Vangl2 (green, arrowheads) in the $\mathrm{OHCS}(01-03)$ and the more extended localization in the IPs (asterisk). L, In situ hybridization with a probe for Vangl2 showing expression of mRNA (purple) in both HCs and SCs in the $O C$ from an E18.5 mouse. The deeper purple color in the IP (asterisk) is probably a result of a relatively higher level of Vang/2 expression in these cells. $\boldsymbol{M}, \mathbf{N}$-cadherin (red) specifically labels IHC membranes, demonstrating a colocalization with Vangl2 (green) on the proximal (arrowheads) and distal (arrows) sides of the IHC in a P2 rat cochlea. $N$, An antibody against $\beta 1 / \beta 2$ tubulin (red) labels the lumenal surfaces of all supporting cells, suggesting a colocalization of Vangl2 (green) at the junction of HCs (asterisks) and supporting cells (arrows). Scale bars: $C$ (for $\boldsymbol{C}-\boldsymbol{F}), \boldsymbol{G}, \boldsymbol{J}, \boldsymbol{N}($ for $\boldsymbol{M}, \boldsymbol{N}), 10 \mu \mathrm{m} ; \boldsymbol{H}$ (for $\boldsymbol{H}, \boldsymbol{I}), \boldsymbol{K}$ (for $\boldsymbol{K}, \boldsymbol{L}), 5 \mu \mathrm{m}$. 
tation on HEK293 cells were performed as described previously after $48 \mathrm{~h}$ transfection with the various cDNA using Lipofectamine 2000 (Invitrogen), according to the protocol of the manufacturer.

Glutathione S-transferase binding assays. The Scrb1-glutathione $S$-transferase (GST) fusion proteins containing PDZ1 (postsynaptic density-95/Discs large/zona occludens-1) (base pairs 710-810), PDZ2 (base pairs 845-940), PDZ3 (base pairs 985-1085), PDZ4 (base pairs 10851185), PDZ1-PDZ2 (base pairs 710-940), PDZ3-PDZ4 (base pairs 9851185), PDZ1-PDZ4 (base pairs 710-1185), and leucine-rich repeat (LRR) plus linker (base pairs 3-714) were expressed in BL21 using pGEX-4T-1 and purified directly from bacterial extract on glutathioneSepharose-4B beads. A pull-down assay was performed with recombinant GFP-Vangl2 subcloned into the pcDNA3.1 and overexpressed in HEK293. A stop codon was introduced by direct mutagenesis to generate a mutant lacking the last four amino acids $\left(\operatorname{Vangl} 2^{\Delta 4}\right)$. A total of $10 \mu \mathrm{g}$ of immobilized GST or specific GST-Scrb1 fusion proteins were incubated with $1 \mathrm{ml}$ of cell lysate expressing recombinant Vangl2 or Vangl2 ${ }^{\Delta 4}$ for $2 \mathrm{~h}$ at $4^{\circ} \mathrm{C}$. After extensive washes with binding buffer, the bead pellets were resuspended in SDS sample buffer and subjected to SDS-PAGE and immunoblotting.

\section{Results}

Vangl2 is asymmetrically localized in the mammalian inner ear

Vangl2 was the first gene to be identified as a PCP gene in the mammalian inner ear (Montcouquiol et al., 2003), and PCP defects in Vangl2 mutants are more severe than in any other currently identified single PCP mutant (Curtin et al., 2003; Lu et al., 2004). Therefore, we raised a peptide antibody against the putative intracellular N-terminal domain of Vangl2. The antibody reacted strongly with an $\sim 65 \mathrm{kDa}$ protein band with lysate from HEK293 cells transfected with Vangl2, as well as in cochlear and brain extracts (Fig. $1 B$ ). The amino acid sequence of mammalian Vangl2 predicts a molecular weight of $59.7 \mathrm{kDa}$, and the Vangl2 protein migrates at an apparent molecular weight of $65 \mathrm{kDa}$ on a $7-20 \%$ gradient SDS polyacrylamide gel. The antibody also recognized a GFP-tagged version of the Vangl2 protein when expressed in various cell lines, including COS1, MDCK, and NRK (data not shown).

The first indications of polarization within the cochlea can be observed in the proximal (inner hair cell) region of the epithelium (Fig. 1C-F) beginning in the rat at approximately E18.5. Confocal images taken at this time point revealed that Vangl2 is membrane associated in most of the cells in the sensory and the nonsensory epithelium (Fig. $1 C-F$ ) (supplemental Fig. 1A, $C$, available at www.jneurosci.org as supplemental material). A punctate staining was also observed in the cytoplasm of most cell types. Within the sensory epithelium, analysis of the distribution of Vangl2 in the most immature region (Fig. 1C,D) indicated asymmetric expression in IPh cells on both the proximal (arrowheads) and distal (arrows) edges of the cells. No noticeable asymmetric accumulation of protein was observed in the unpolarized outer hair cell region of the epithelium at this time point (brackets). In a more differentiated region of the cochlea at the same time point, asymmetric localization of Vangl2 in IPh cells (Fig. $1 E, F$, arrowheads) (supplemental Fig. $1 B, D$, available at www. jneurosci.org as supplemental material) was more intense. In addition, asymmetric localization of Vangl2 had now extended to the next adjacent cells, inner and outer pillar cells (white arrows). IHCs (asterisks) also showed an asymmetric accumulation of Vangl2 on their proximal edges. In contrast, the accumulation of Vangl2 on the distal edges, the edges in contact with pillar cells, was negligible. Finally, some asymmetric localization of Vangl2 was also observed on the first and second rows of developing outer hair cells (Fig. 1E,F, yellow arrows). As was observed for inner hair cells, Vangl2 accumulation in hair cells was restricted to the proximal edge.

With continued development of PCP within the OC, asymmetric localization of Vangl2 sharpened within individual hair cells and supporting cells. At P1, in rat, we observed a strong expression of Vangl2 at the junction between the proximal edge of developing hair cells and the distal edges of adjacent supporting cells (Fig. 1G) (supplemental Fig. $1 E$, available at www. jneurosci.org as supplemental material). Single-plane confocal analysis indicates that each $\mathrm{OHC}(\mathrm{O} 1, \mathrm{O} 2, \mathrm{O} 3)$ contacts four or five SCs, one or two on its proximal edge (depending on position), one on each of its lateral sides, and one on its distal edge (Fig. $1 H, I$ ). However, Vangl 2 only accumulates on the proximal boundary of each $\mathrm{OHC}$. These accumulations points directly correlate with the plane of polarization, suggesting that an active process must restrict Vangl2 to these junctions. A similar pattern of Vangl2 localization was observed in P0 mouse cochlea (Fig. $1 \mathrm{~J})$. In cross-section, the accumulation of Vangl2 was clearly restricted to the lumenal regions of OHCs (Fig. $1 \mathrm{~K}$, arrowheads). In contrast, a more basal extension of Vangl2 that extended $\sim 6.5$ $\mu \mathrm{m}$ from the lumenal surface was observed in IPs (compared with a basal extension of only $1.2 \mu \mathrm{m}$ in hair cells) (Fig. $1 \mathrm{~K}$, asterisk). Moreover, in situ hybridization on an E18.5 mouse cochlea indicates that mRNA for Vangl2 is expressed in hair cells and supporting cells (Fig. $1 \mathrm{~L}$ ), with a higher level of expression in pillar cells (Fig. $1 L$, asterisk).

At time points that extended beyond the primary period of polarization, expression of Vangl2 protein appeared negligible in most cell types (supplemental Fig. 2, available at www.jneurosci.org as supplemental material). However, we were able to detect some Vangl2 protein in the lumenal surfaces of the pillar cells (supplemental Fig. 2A, $B$, available at www.jneurosci.org as supplemental material) and on the proximal sides of the outer hair cells in the apex of a P7 cochlea. Analysis of more mature regions suggests that that expression of Vangl2 does not persist beyond the early postnatal period in the sensory region (supplemental Fig. 2C,D, available at www.jneurosci.org as supplemental material)

\section{Vanlg2 is asymmetrically localized in hair cells and supporting cells in the OC}

Vangl2 becomes clearly asymmetrically localized to the boundaries between hair cells and supporting cells, but, because cell borders were identified by detection of actin or $\beta$-catenin, both of which are present in both hair cells and supporting cells, it was not possible to determine whether Vangl2 was asymmetrically located in HCs, SCs, or both. Therefore, Vangl2 was colocalized with cell-type-specific markers in an effort to determine whether the distribution of Vangl2 varies with cell type. N-cadherin has been shown to stain the membranes of inner hair cells but not those of any other cell types in the OC (Simonneau et al., 2003). $\mathrm{N}$-cadherin and Vangl2 colocalized at the membrane of the inner hair cells, both distally (Fig. $1 M$, arrow) (supplemental Fig. $3 A$ ) and proximally (Fig. $1 M$, arrowheads) (supplemental Fig. $3 A$, available at www.jneurosci.org as supplemental material). Next, Vangl2 was colocalized with $\beta 1 / \beta 2$-tubulin, a protein that is strongly expressed in SCs of the OC (Hallworth et al., 2000). Staining indicated asymmetric accumulation of Vangl2 at the distal edges of different SC types, suggesting that Vangl2 is asymmetrically localized in both HCs (Fig. $1 \mathrm{~N}$, asterisks) (supplemental Fig. $3 B$, available at www.jneurosci.org as supplemental material) and SCs (Fig. $1 \mathrm{~N}$, arrows). 

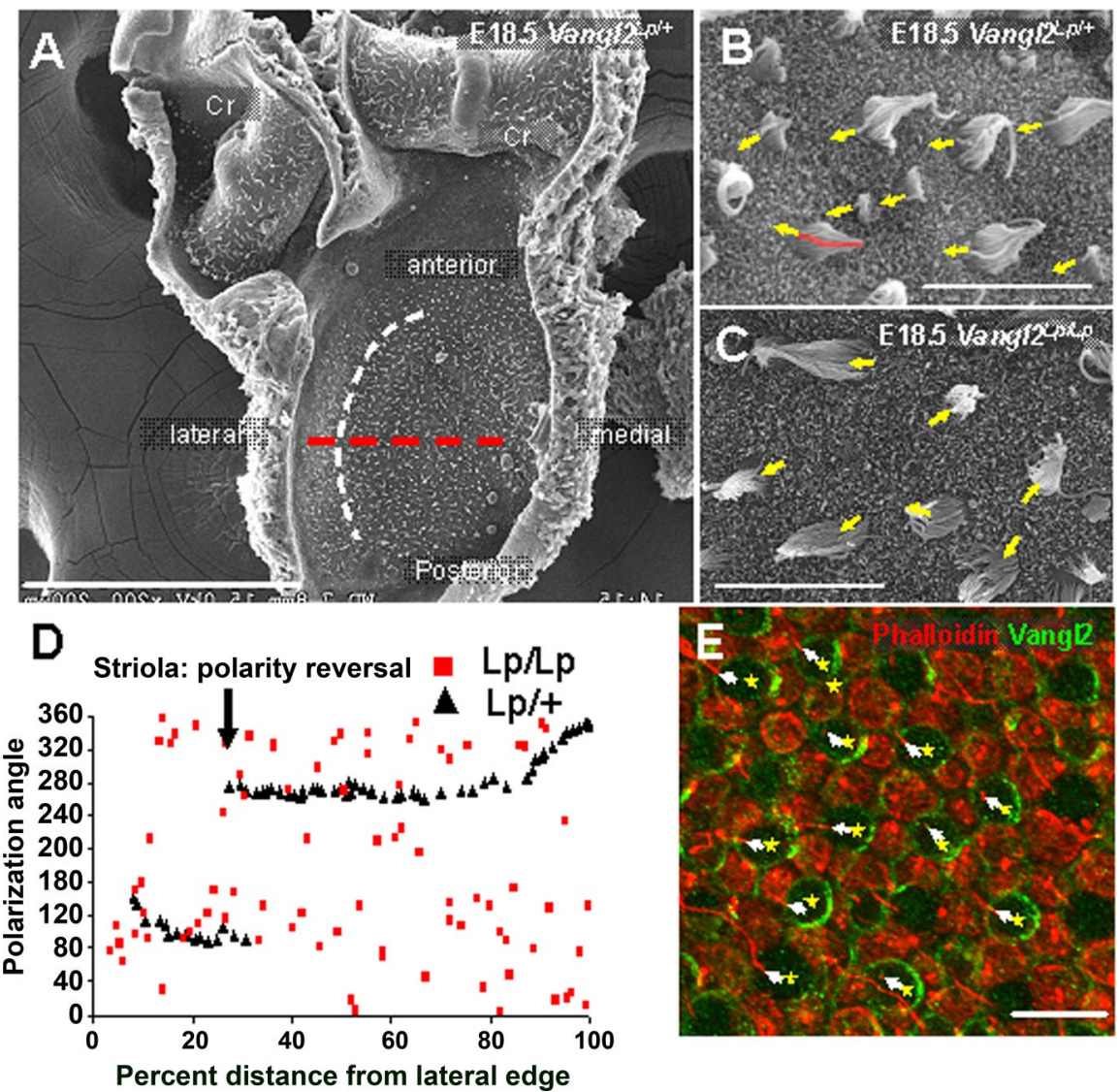

Figure 2. $P C P$ is disrupted in the vestibular system of $V a n g / 2^{L p / L p}$ animals. $A$, Scanning electronic micrograph of the surface of a utricle from a Vangl2 ${ }^{L /+}$ animal atE18.5, illustrating the flat aspect of the tissue, compared with the two cristae (Cr) that are still attached. The surface of the utricle is covered with the stereociliary bundles of the hair cells. The striolar reversal zone is illustrated by the white curved dotted line. Hair cells located on both sides of the striola are oriented such that kinocilium on each bundle points toward the striola. See $\boldsymbol{D}$. The red dotted line indicates mediolateral axis used to obtain hair cell polarization angles in $\boldsymbol{D}$. $\boldsymbol{B}$, $\boldsymbol{C}$, High-magnification images of stereociliary bundles in utricles from Vangl $2^{L /+}(\boldsymbol{B})$ and $\operatorname{Vang} / 2^{L p / L p}(\boldsymbol{C})$ animals. Bundle orientations are indicated by arrows based on the position of the kinocilium in each hair cell (red pseudocolor on the bundle at the extreme left edge of the image in $\boldsymbol{B}$ ). $\boldsymbol{D}$, Scatter plot of stereociliary bundle orientations along a line extending along the mediolateral axis of the utricle perpendicular to the striola (red dotted line in $\boldsymbol{A}$ ). In the utricle from the Vangl $2^{L /+}$ animal, stereociliary bundles located lateral to the striola are oriented with the kinocilium on the side of the cell closest to the striola (polarization angle of $90^{\circ}$ ). Medial to the striolar reversal, there is a $180^{\circ}$ polarity reversal, resulting in a polarization angle of $270^{\circ}$. In contrast, bundle orientations in a utricle from a $\operatorname{Vang} / 2^{L p / L p}$ animal appear to be randomly distributed, demonstrating a severe PCP defect. $\boldsymbol{E}$, Luminal surface of a WT PO utricle labeled with $\beta 1 / \beta 2$-tubulin (red) and Vangl2 (green). The yellow asterisks mark the position of the hair cells, and the arrows indicate the position of the kinocilium (red), indicating the polarity of each bundle. Vangl2 is asymmetrically expressed on the proximal side of the hair cells, opposite to the kinocilium. Supporting cell membranes also express Vangl2. Scale bars: $\boldsymbol{A}, 200 \mu \mathrm{m} ; \boldsymbol{B}, \boldsymbol{C}, \boldsymbol{E}, 10 \mu \mathrm{m}$.

\section{Vangl2 is asymmetrically localized in hair cells and} supporting cells and regulates PCP in the vestibular system In addition to the cochlea, the inner ear also contains vestibular sensory epithelia that mediate the senses of balance and acceleration. The utricle and saccule, which detect linear acceleration, and the three cristae ampullaris, which detect head rotation, also contain mechanosensory hair cells and nonsensory supporting cells. However, in contrast with the cochlea, hair cells in these epithelia are arranged into large sensory patches (Fig. 2A). To confirm that PCP in these epithelia is also regulated by Vangl2, we analyzed stereociliary bundle orientations in utricles from a single animal that was heterozygous for the looptail mutation (referred to as Vangl2 ${ }^{L p /+}$ ) (Fig. $2 B$ ) and three Vangl2 $2^{L / L p}$ homozygotes at E18.5 (Fig. 2C). The orientation of each stereociliary bundle was determined by using the position of the asymmetrically localized kinocilium (Montcouquiol et al., 2003) (Fig. $2 B, C$, arrows and red pseudocolor). The utricle was selected be- cause this sensory epithelium tends to be flat and easier to study compared with the more curved cristae (Fig. $2 \mathrm{~A}$ ). The utricle contains two populations of hair cells with opposite planes of polarization. There is a reversal zone, referred to as the striola, located $\sim 25 \%$ from the distal edge of the epithelium (Fig. $2 \mathrm{~A}$, white dotted line). A plot of stereociliary bundle orientations along a line extended through the middle of the utricle along the lateromedial axis (Fig. 2A, red dotted line) from the Vangl2 $2^{L /+}$ animal illustrates the two populations of oppositely oriented hair cells and the striolar reversal zone (Fig. 2D, black triangles). In contrast, a similar plot from a Vangl2 ${ }^{L p / L p}$ littermate clearly demonstrates a striking disruption in bundle orientation (Fig. $2 D$, red squares). In fact, an analysis of all of the orientations observed through the entire utricle indicates that the bundle orientations may be randomly distributed. To determine whether Vangl2 becomes similarly localized in vestibular sensory epithelia, Vangl2 was localized in developing hair cells and supporting cells. As expected, Vangl2 was found on the proximal edges of developing hair cells and on the distal edges of supporting cells in a pattern that appeared comparable with that observed in the cochlea (Fig. $2 E$ ).

\section{Asymmetric localization of Vangl2 is lost in Vangl $2^{L p / L p}$ mutants}

Looptail mice carry a point mutation in Vangl2 that causes a serine-to-asparagine transition at amino acid position 646 (referred to as $\operatorname{Vangl2}^{L p}$ ) (Kibar et al., 2001; Murdoch et al., 2001). To begin to examine the nature of the $\operatorname{Vangl} 2^{L P}$ mutation, the localization of Vangl2 in the cochleae of $\operatorname{Vangl2}^{L p / L P}$ was determined. Surprisingly, no Vangl2 protein was detected in any cell types within the cochleae of these animals (Fig. $3 A, B$ ). Previous studies had indicated by in situ hybridization and Northern blot that Vangl 2 mRNA is present in Vang $12^{\mathrm{Lp} / \mathrm{Lp}}$ mice, and that the mutation does not affect the stability of the protein in yeast (Kibar et al., 2001; Murdoch et al., 2001; Torban et al., 2004). To confirm that no Vangl2 protein was present, we used protein extracted from brains to compare the levels between WT and $\operatorname{Vangl2}^{L p / L p}$. As illustrated in Figure 3C, Vangl2 was barely detectable in the sample isolated from $V a n g l 2^{L p / L p}$ brain. This result suggests a posttranslational degradation of $\operatorname{Vangl}^{L P}$ resulting in an effective null mutation of Vangl2. To confirm this hypothesis, HEK293 cells were cotransfected with 4 or $2 \mu \mathrm{g}$ of Vangl2-GFP or Vangl2 ${ }^{L P}$-GFP and an empty DsRed vector. If both proteins are expressed at similar levels, then the level of DsRed should be equivalent in both transfections whereas the level of Vang ${ }^{\text {Lp }}$-GFP should be lower than Vangl2-GFP. As illustrated in Figure $3 C$ (bottom), the level of DsRed was equivalent for both conditions, whereas the level of Vangl2 ${ }^{\mathrm{LP}}$ appears to be reduced by comparison with Vangl2. 
Our data suggested that Vangl2 ${ }^{\mathrm{Lp}}$ might not be targeted or anchored to the membrane, and, as a result, targeted for degradation. We tested this hypothesis in epithelial MDCK cells. When a Vangl2GFP fusion construct was transfected into MDCK cells, the protein was targeted to the membrane of the cells as expected (Fig. $3 D, E)$, with a strong accumulation of the protein at the junction between two transfected cells (Fig. 3E, arrows). In contrast, a Vangl $2{ }^{\mathrm{Lp}}$-GFP fusion protein was no longer targeted to the membrane when expressed in the same cell line and, instead, appeared to accumulate in the cytoplasm of the cells (Fig. 3F, G). These data suggest that the serine at position 464 is important for membrane targeting and/or anchoring and that, in the absence of this signal, the protein is degraded.

To determine whether the Vangl2 ${ }^{L P}$ mutation has a similar effect on Vangl2 localization in the cochlea, Vangl2-GFP and $\operatorname{Vangl2}^{L P}{ }_{-G F P}$ vectors were transfected in rat cochlea at E14.5, a time point that correlates with the onset of $\mathrm{HC}$ and SC development. As expected, Vangl2-GFP protein was localized at the membrane in both HCs and SCs (Fig. $3 \mathrm{H}, \mathrm{I}$ ). In contrast, Vangl2 ${ }^{\mathrm{LP}}$-GFP protein was not observed at the membrane and instead remained within the cytoplasm (Fig. 3 J,K). The lack of asymmetric protein localization in cells transfected with Vangl2-GFP could be the result of the strong expression generated by the CMV promoter and a subsequent accumulation of protein saturating the regulatory mechanisms controlling the asymmetry.

Scrb1 physically interacts with Vangl2 and is required for asymmetric membrane targeting of Vangl2

Scrb1 was demonstrated recently to act as a PCP gene in the cochlea and to interact at a genetic level with Vangl2 (Montcouquiol et al., 2003). Immunolocalization for Scrb1 indicated expression in all cell types within the OC (Fig. 4A). In addition, the protein was localized along the basolateral membranes of HCs and SCs (Fig. 4B), as reported previously (Navarro et al., 2005). In MDCK cells, endogenous Scrb1 is strongly expressed at the cell membranes, in which Vangl2 is targeted when overexpressed (Navarro et al., 2005; Qin et al., 2005). Therefore, potential direct interactions between Scrb1 and Vangl2 were examined. First, a yeast two-hybrid screen using a portion of the C-terminal part of Vangl2, including the PDZ binding domain, as bait, isolated Scrb1 from a mouse E17 embryonic library (data not shown). To confirm the requirement of the PDZ binding domain, coimmunoprecipitation of Vangl2 and Scrb1 was performed through cell transfections. HEK293 H-K), $20 \mu \mathrm{m}$.
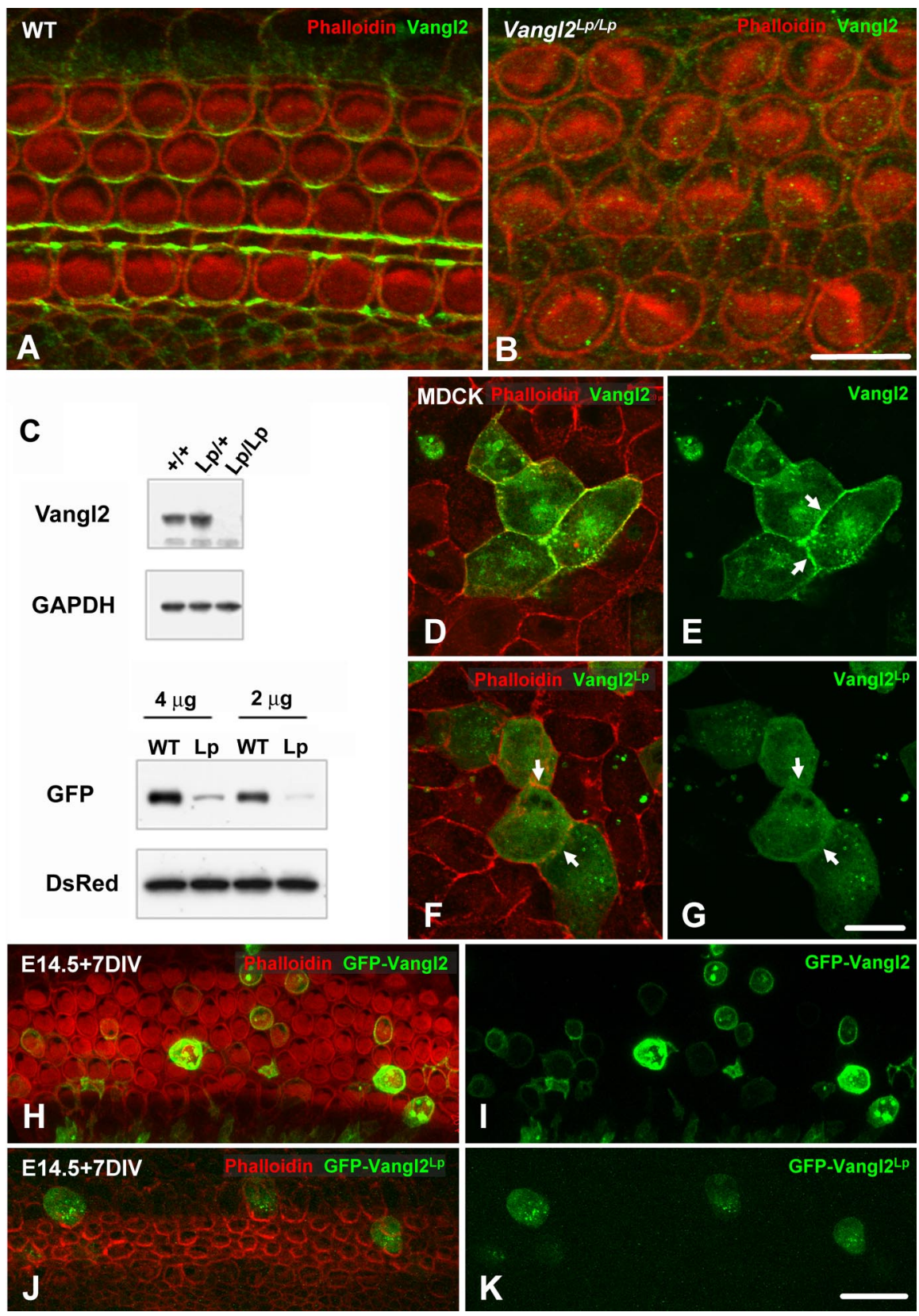

Figure 3. Vangl2 is absent in Vang $\left.\right|^{L / L p}$ mice. $\boldsymbol{A}, \boldsymbol{B}$, Lumenal surface of the $0 C$ from E18.5 WT $(\boldsymbol{A})$ and $V a n g l 2^{L p / L p}(\boldsymbol{B})$ littermates. Cell boundaries are labeled with phalloidin (red), and Vangl2 expression is in green. Vangl2 expression is completely absent in the cochlea from a Vangl $2^{L p / L p}$ littermate. C, Top, Brain lysates from WT $(+/+$, line 1$)$, heterozygous $(L p /+$, line 2$)$, and homozygous (Lp/Lp, line 3) Vang ${ }^{L p}(L p)$ mice were analyzed by immunoblotting. Vangl2 protein was expressed in brains from WT and heterozygotes but absent in brain from homozygotes, suggesting a degradation of the protein in the mutant. $\mathbf{C}$, Bottom, HEK293 cells were cotransfected with different levels of GFP-Vangl2 (WT) or GFP-Vangl2 ${ }^{L p}$ (Lp) and an empty DsRed vector. The immunoblot probed with an anti-GFP antibody reveals that the expression level of Vangl2 ${ }^{\mathrm{Lp}}$ is weaker than that of Vangl2, whereas the same amount of transfected protein is present in the sample, as indicated by the levels of DsRed. D, E, Vangl2 is targeted to the membrane of transfected MDCK cells, with a strong accumulation at the junction between two transfected cells (arrows).F, G, By comparison, the Vangl ${ }^{\mathrm{Lp}}$ construct is no longer targeted to the cell membrane (arrows) and accumulates in the cytoplasm of the cells. $\boldsymbol{H}-\boldsymbol{K}$, Lumenal surfaces of E14.5 rat cochleae, electroporated with either Vangl2 $(\boldsymbol{H}, \boldsymbol{I})$ or Vangl2 ${ }^{\mathrm{Lp}}(\boldsymbol{J}, \boldsymbol{K})$ and maintained for $7 \mathrm{~d}$ in vitro (DIV). Whereas Vangl2 (green) was strongly expressed and targeted to the cell membrane, Vangl2 ${ }^{\mathrm{Lp}}$ accumulated in the cytosol of the cells, with lower levels of expression. Scale bars: $\boldsymbol{B}($ for $\boldsymbol{A}, \boldsymbol{B}), 10 \mu \mathrm{m} ; \boldsymbol{G}($ for $\boldsymbol{D}-\boldsymbol{G}), \boldsymbol{K}($ for

cells were cotransfected with Vangl2-GFP and Scrb1, and we used anti-GFP antibodies to coimmunoprecipitate Scrb1 (Fig. 4C, lane 5). This interaction was strongly reduced with Vangl ${ }^{\mathrm{Lp}}$ (Fig. $4 C$, lane 6) and abolished in the absence of the PDZ binding domain on Vangl2 (Vangl2 ${ }^{\Delta 4}$ ) (Fig. $4 C$, lane 7 ). Anti-GFP antibodies 

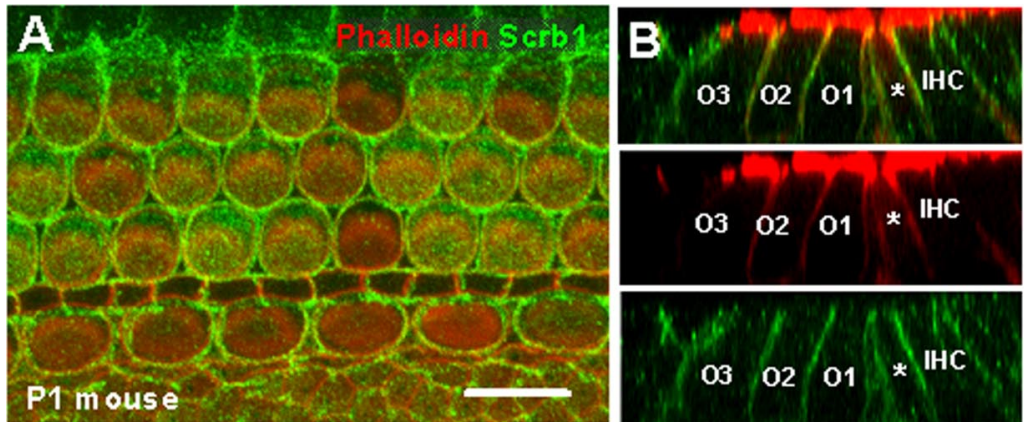

\section{C}
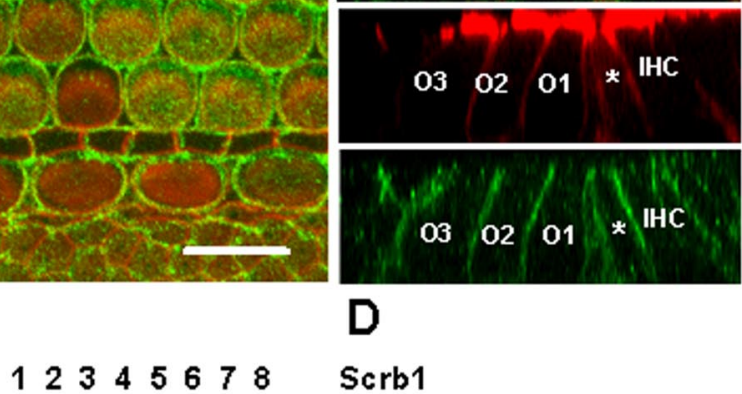

D

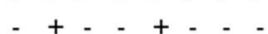

$-+-{ }_{-}^{-}+\cdots$

$-++-+c_{-}^{-}+$

$+-{ }_{-}++\cdot$

GFP.Vangl2 4

Scribble1
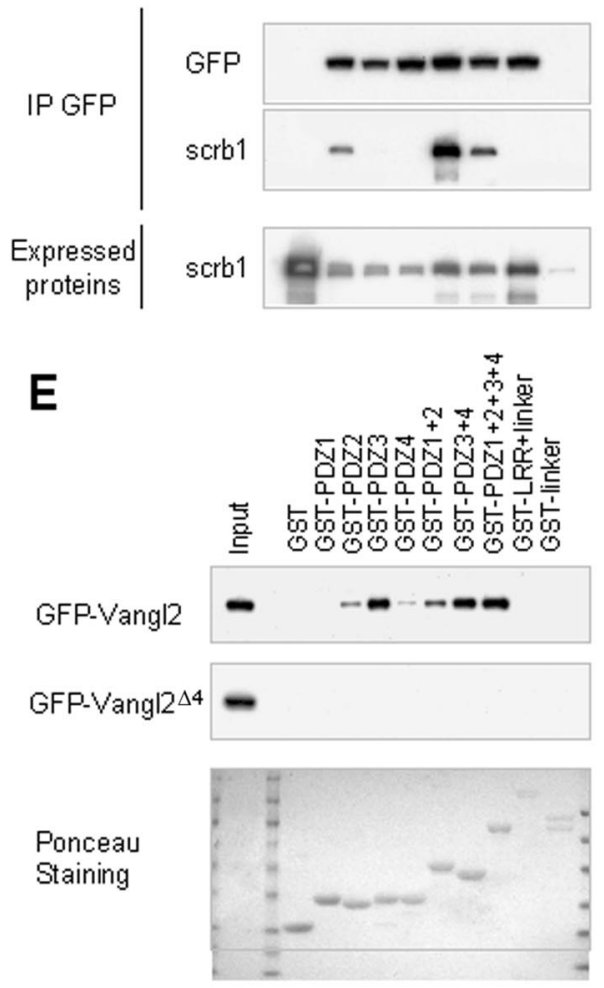
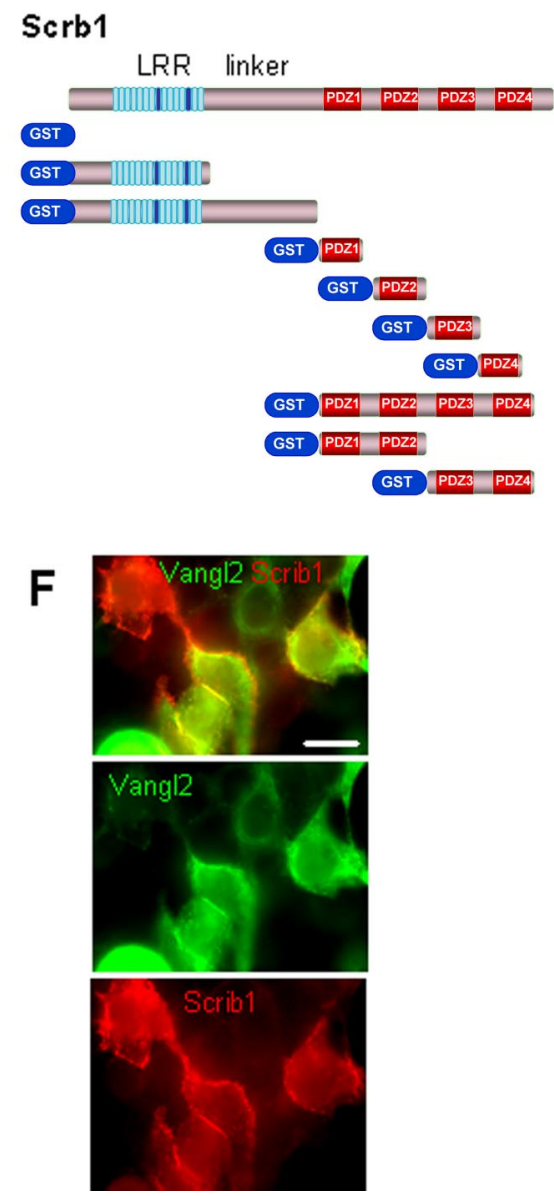

Figure 4. Scrb1 and Vangl2 physically interact, and Scrb1 is required for Vangl2 asymmetry. $\boldsymbol{A}$, Lumenal surface of a P1 mouse cochlea labeled with an antibody against Scrb1 (green) indicates widespread expression of the protein at the membranes of all the cells in the OC. $\boldsymbol{B}$, Confocal Z-stack cross-section of a P1 mouse cochlea labeled as in $\boldsymbol{A}$. Top illustrates merged image, and middle and bottom indicate individual phalloidin (red) and Scrb1 (green) channels. Scrb1 is distributed along the entire basolateral membranes of the IHCs and OHCs (numbered) as well as the IPs (asterisk). C, Immunoprecipitation of Scrb1 with Vangl2. HEK293 cells were transfected with Scrb1 and GFP-Vangl2 or GFP-Vangl2 ${ }^{\mathrm{Lp}}$ constructs, and proteins were immunoprecipitated with an anti-GFP antibody. The membranes were then probed with our anti-Scrb1 antibody. The presence of a band indicates that Vangl2 and Vangl2 ${ }^{\text {Lp }}$ can interact with Scrb1 (lanes 5, 6), but removal of the PDZ binding domain at the C terminus of Vangl2 (Vangl2 ${ }^{\Delta 4}$ ) prevents this interaction (lane 7). The band in column 2 represents endogenously expressed Scrb1 in HEK293 cells. D, Structure of mammalian Scrb1, with an LRR near the $\mathrm{N}$ terminus, a linker region, and four PDZ domains at the C terminus. Beneath the full structure is a graphical list of the GST constructs used in the GST pull-down assay in $\boldsymbol{E}$. $\boldsymbol{E}$, GST pull downs indicate that Vangl2 can interact with PDZ2, PDZ3, or PDZ4, as well as other combinations of PDZ domains from Scrb1, with the strongest interaction with PDZ2 and PDZ3. In contrast, VangI2 does not interact with the LRR or linker domains. Mutation of Vangl2 on serine S464 leads to a strong reduction in Scrb1 binding, and mutation of the last four amino acids of Vangl2 (Vangl2 ${ }^{\Delta 4}$ ) completely suppresses this binding. $\boldsymbol{F}$, Immunolocalization of Vangl2 and Scrb1 in HEK293 cells. HEK293 cells were transfected with Scrb1 (red) and GFPVangl2 (green) constructs, and proteins were processed for immunocytochemistry with anti-GFP and anti-Scrb1 antibodies. The two proteins colocalize strongly when coexpressed in the cells. Scale bar, $10 \mu \mathrm{m}$.

failed to immunoprecipitate Scrb1 in the absence of Vangl2-GFP, demonstrating that Vangl2 interacts specifically with Scrb1 in mammalian cells. Nonimmune IgGs did not immunoprecipitate either Vangl2 or Scrb1 (data not shown).

Scrb1 contains an N-terminal LRR and four PDZ domains located at the C-terminal end, separated by a linker region (Fig. 4D). To map the interacting domains of Scrb1 with Vangl2, individual PDZs as well as PDZ1-PDZ2 and PDZ3PDZ4 combinations were assayed for their ability to independently bind to GFPtagged Vangl2 (Fig. 4D). With the exception of the first PDZ domain (PDZ1), all PDZ domains of Scrb1 (PDZ2, PDZ3, and PDZ4), as well as the combinations of PDZ1-PDZ2 or PDZ3-PDZ4 were able to bind Vangl2 (Fig. 4E). PDZ2 and PDZ3 bind to Vangl2 with the strongest affinity. Constructs including the N-terminal part of Scrb1, including just the LRR, the LRR with the linker, or GST alone were not able to bind to Vangl2. When we removed the PDZ binding domain of Vangl2 (Vangl2 ${ }^{\Delta 4}$ ), the interaction with Scrb1 was lost (Fig. 4E). Finally, HEK293 cells cotransfected with expression vectors for both Scrb1 and Vangl2 exhibited a colocalization of the proteins (Fig. $4 F$ ).

The demonstrated physical interactions between Scrb1 and Vangl2 suggested a role for Scrb1 in targeting of Vangl2. Therefore, the asymmetric localization of Vangl2 was examined in cochleae from

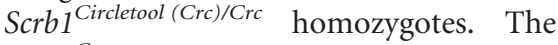
$S c r b 1{ }^{C r c}$ mutation is a truncating mutation that leads to the loss of the last two PDZ domains, PDZ3 and PDZ4. Despite the mutation in Scrb1, Vangl2 was still present at the membranes of both HCs and SCs in cochleae from Scrb1 $1^{\mathrm{Cr} / \mathrm{Crc}}$ homozygotes (Fig. 5A, B). However, the asymmetric distribution of Vangl2 was significantly disrupted (Fig. 5B). An analysis of the distribution of Vangl2 at specific points along the luminal-basal axis of the epithelium indicated a nearly complete disruption in the asymmetric distribution of the protein in HCs and SCs located in outer hair cell rows 2 and 3 but a preservation of asymmetry in IPh and pillar cells (Fig. 5C-F, arrows). The loss of Vangl2 asymmetry, in particular in the second and third rows of $\mathrm{OHC}$, strongly correlates with the more pronounced PCP defects in this region of Scrb1 ${ }^{\mathrm{Crc} / \mathrm{Crc}}$ cochleae (Montcouquiol et al., 2003).

\section{Celsr 1 is required for asymmetric localization of Vangl2}

Celsr1, a vertebrate homolog of the core $\mathrm{PCP}$ gene Flamingo, is required for PCP in 

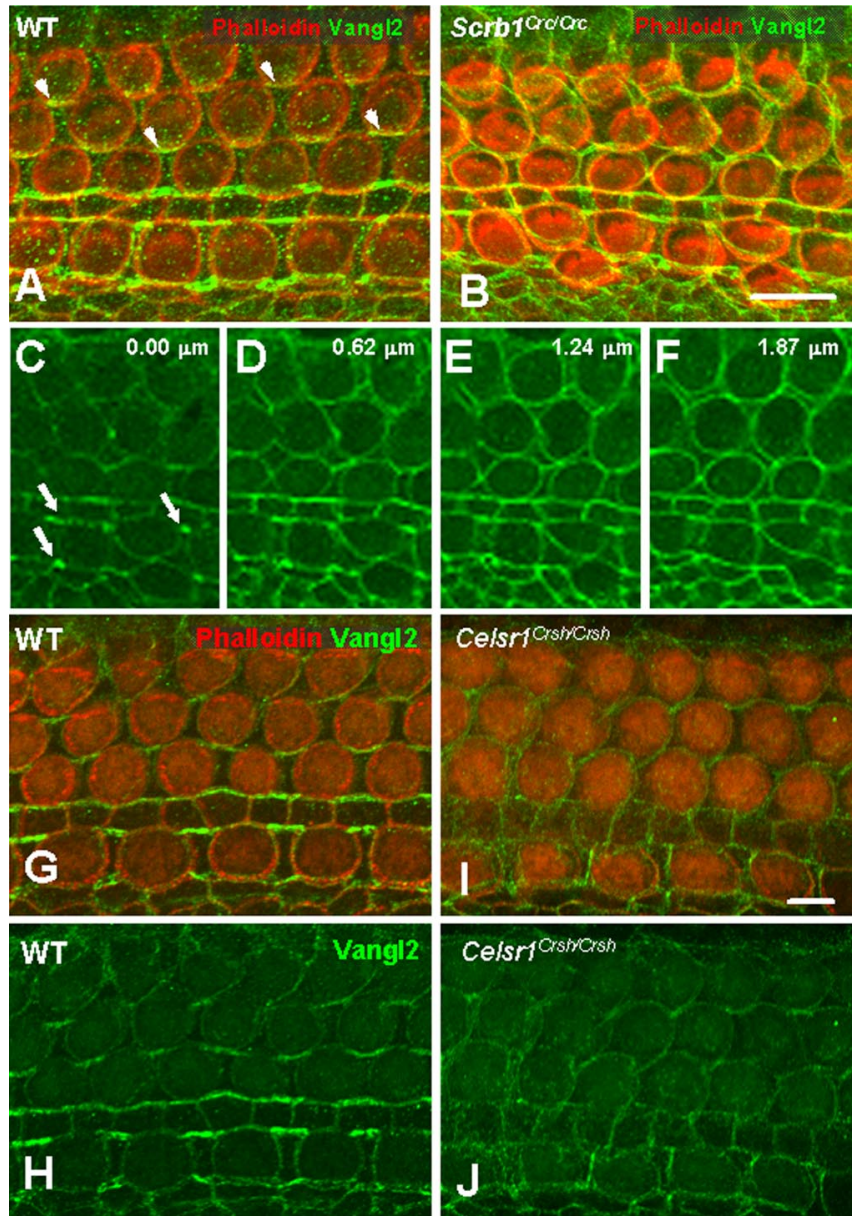

Figure 5. Vangl2 asymmetry is lost in $\mathrm{Scrb}_{1} 7^{\mathrm{Cr} / \mathrm{Cr} c}$ and Celsr $7^{\mathrm{Crsh} / \mathrm{rsh} h}$ mutants. $\boldsymbol{A}, \boldsymbol{B}$, Lumenal surface of the 0 C from an E18.5 WT (A) and Scrb $7^{\text {Cre/ } / \text { re }}$ littermate. In contrast with WT $(\boldsymbol{A})$, Vangl2 (green) asymmetry is lost in cochleae from $\mathrm{Scrb}_{1}{ }^{\mathrm{Cr} /(\mathrm{rr} c}$ mutants $(\boldsymbol{B})$, but the protein is still targeted to the membrane. $\boldsymbol{C}-\boldsymbol{F}$, The same image as in $\boldsymbol{B}$ but with only the green channel, at four different focal planes, beginning at the lumenal surface and advancing into the tissue by $0.62 \mu \mathrm{m}$ in each frame. In contrast with $\mathrm{OHCs}$, a persistent asymmetric localization of Vangl2 can be observed in the IPh cells and pillar cells near the lumenal surface (arrows in $C$, but a nearly uniform distribution of the protein is present in all cells at deeper focal planes $(\boldsymbol{D}-\boldsymbol{F})$.

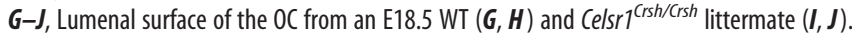
Vangl2 alone is illustrated in $\boldsymbol{H}$ and $\boldsymbol{J}$. In contrast with WT, there is an overall decrease of Vangl2 (green) at the membrane and a uniform distribution of the protein in the $0 \mathrm{C}$ from the Celsr $7^{\text {(rsh/ }}$ crsh animal. Scale bars: $\boldsymbol{A}$ (for $\boldsymbol{A}, \boldsymbol{B}), \boldsymbol{I}($ for $\mathbf{G}-\boldsymbol{J}), 10 \mu \mathrm{m}$.

the mammalian cochlea and is asymmetrically localized to both proximal and distal membranes during development of PCP in the chicken cochlea (Davies et al., 2005). The role of Celsr1 in asymmetric localization of Vangl2 was examined by analyzing Vangl2 asymmetry in cochleae from Celsr $1^{\mathrm{Crash}(\mathrm{Crsh}) / \mathrm{Crsh}}$ mutants. Although Vangl2 is still targeted to the membranes of HCs and SCs in Celsr1 $1^{\text {Crsh/Crsh }}$ mutants, there is a nearly complete loss of asymmetry and a decrease in the overall level of Vangl2 protein at the membrane (Fig. 5G-J), suggesting that Celsr1 is required for the recruitment and asymmetric localization of Vangl2.

\section{Fz3 is asymmetrically localized in a pattern similar to Vangl2}

$\mathrm{Fz}$ proteins have been shown to act as the upstream receptors for the PCP signaling cascade (Adler and Lee, 2001; Strutt, 2003), and $F z 3 / 6$ double mutants have PCP defects in the cochlea (Wang et al., 2006). Therefore, we used a commercially available antibody against Fz3 to determine its localization in the cochlea. Fz3 was asymmetrically localized to the proximal edges of hair cells in a pattern similar to that of Vangl2 at the same developmental time point (Fig. 6A,B). In addition, analysis of cross-sections indicated that $\mathrm{Fz} 3$ was also restricted to the apical region of each hair cell (Fig. 6C-E). Because both antibodies were generated in rabbits, it was not possible to confirm colocalization because of cross-reactivity at the level of the secondary antibodies, but the results strongly suggest that possibility. To determine whether localization of Vangl2 plays a role in the generation of Fz3 asymmetry, the expression of Fz3 was examined in cochleae from Vangl $2^{L p / L p}$ homozygotes. In these mutants, we observed a complete loss of Fz3 at the membrane, suggesting a role for Vangl2 in Fz3 targeting or maintenance at the membrane (Fig. $6 F-I$ ). An interaction through Scrb1 was tested, but Fz3 did not interact with Scrb1 (data not shown). Based on these results, potential direct interactions between Vangl2 and Fz3 were determined by immunoprecipitation. As expected, a direct interaction between Vangl2 and Fz3 was demonstrated in HEK293 cells transfected with untagged Vangl2 and Fz3-GFP (Fig. 6J). The two bands observed result most probably from monomer and dimer forms of Fz3.

\section{Discussion}

Vangl2 plays a key role in polarization of $\mathrm{HC}$ within the mammalian cochlea. The results presented here suggest that asymmetric localization of Vangl2 to cell-cell boundaries provides crucial instructive interactions that direct the overall polarization of the individual cells within the epithelium. Overall, our data suggest conservation of some aspects of the PCP signaling cascades, but the results also indicate that major differences exist between the vertebrate and invertebrate PCP signaling cascades. In particular, although the results presented here demonstrate that Fz3 is asymmetrically localized as in Drosophila, in the cochlea, Fz3 and Vangl2 interact and become localized to the same region of the cell. These results contrast strikingly with results from Drosophila wing in which vang/strabismus (stbm) and fz are specifically localized to opposite sides of a single cell (Adler, 2002; Strutt, 2002).

\section{Asymmetric localization of Vangl2 is required for PCP in the mammalian cochlea}

In Drosophila, after their initial symmetric apical localization, specific PCP proteins become asymmetrically enriched across the respective boundaries along the proximodistal axis in the wing or the dorsoventral axis in the eye (Uemura and Shimada, 2003; Fanto and McNeill, 2004). In particular, vang/stbm becomes localized to the proximal edge of developing wing cells (Fig. 7). Although several models have been proposed to explain the significance of this asymmetry, the specific cellular effects remain unclear (Klein and Mlodzik, 2005). The localization of Vangl2 in developing hair cells is comparable with Drosophila wing, with a restricted accumulation at the proximal boundary of each cell. Moreover, the timing of this asymmetry immediately precedes the first indications of cellular polarization, the centrifugal migration of the developing kinocilium from the center of the lumenal surface of each developing hair cell toward the peripheral edge (Montcouquiol et al., 2003). The direction of this migration is nonrandom and is mediated, in part or in full, depending on HC location, by Vangl2. Based on these results, it seems likely that a key step in this process is the restriction of Vangl2 to the proximal side of each hair cell, suggesting that this restriction either directly or indirectly establishes the directional vector for the subsequent centrifugal migration.

This hypothesis is strongly supported by the observation that there is a loss of Vangl2 asymmetry in both Celsr1 ${ }^{\text {Crshh/Crshh }}$ and 

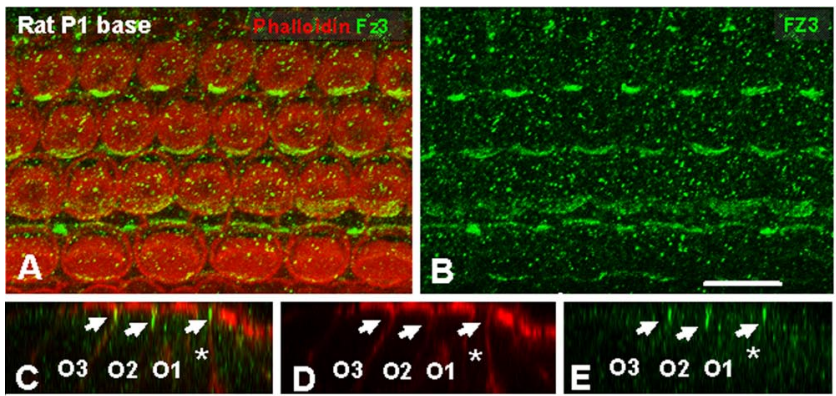

$\mathbf{J}$
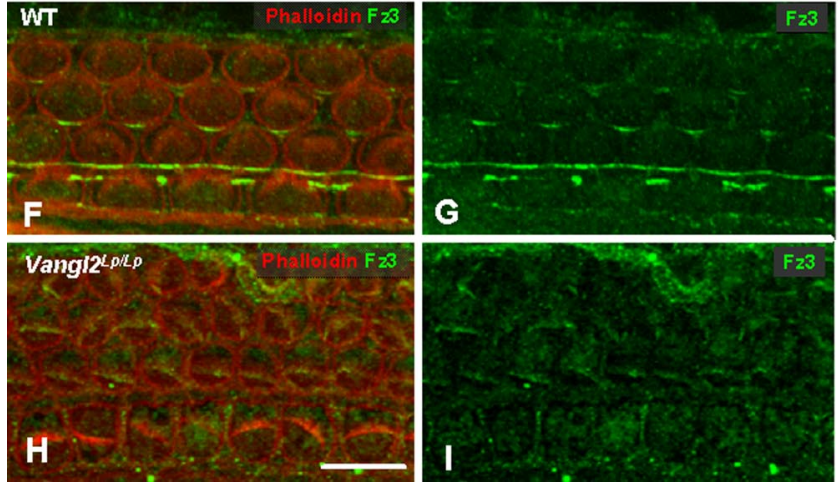

Figure 6. $\quad \mathrm{Fz} 3$ is asymmetrically localized in the 0 C through interactions with Vangl2. $\boldsymbol{A}, \boldsymbol{B}, \mathrm{Fz} 3$ (green) is asymmetrically distributed in a P1 rat cochlea, with a pattern similar to Vangl2. $\boldsymbol{C}-\boldsymbol{E}$, Z-stack confocal cross-section labeled as in $\boldsymbol{A}$, in a P1 rat cochlea. Fz3 (green) is localized to the apical region of two $0 \mathrm{HCs}(02,03)$ and to the IP (asterisk). $\boldsymbol{F}-\boldsymbol{I}$, In contrast with WT $(\boldsymbol{F}, \boldsymbol{G})$, Fz3 (green) asymmetry is lost in E18.5 cochleae from Vangl $2^{L p / L p}$ mice $(\boldsymbol{H}, \boldsymbol{I})$. In fact, Fz3 appears to be entirely absent from the cell membranes. J, Vangl 2 immunoprecipitates with Fz3 in lysates from transfected HEK293 cells (lane 4). The blot shows the presence of two bands, corresponding to the monomeric and dimeric forms of Fz3. Scale bars: $\boldsymbol{B}$ (for $\boldsymbol{A}, \boldsymbol{B}), \boldsymbol{H}$ (for $\boldsymbol{F}-\boldsymbol{I}$ ), $10 \mu \mathrm{m}$.

Scrb1 ${ }^{\mathrm{Crc} / \mathrm{Crc}}$ mutants. In particular, in $\mathrm{Scrb} 1^{\mathrm{Crc} / \mathrm{Crc}}$ mutants, Vangl2 asymmetry is lost in OHCs located in rows 2 and 3, whereas the asymmetric distribution of Vangl2 is maintained in supporting cells in the row of IHCs and first row of OHCs. The loss of Vangl 2 asymmetry in $\mathrm{OHC}$ rows 2 and 3 correlates exactly with the disruption of PCP in $\mathrm{Scrb1} 1^{\mathrm{Crc} / \mathrm{Crc}}$, which is also restricted to $\mathrm{OHC}$ rows 2 and 3 (Montcouquiol et al., 2003). Similarly, there is a more widespread loss of Vangl2 asymmetry in Celsr1 ${ }^{\text {Crsh/Crsh }}$ that correlates with a greater degree of disruption in PCP (Curtin et al., 2003). Finally, a comparison of the severity of PCP defects in mutations in which Vangl2 asymmetry is lost ( $\mathrm{Scrb1} 1^{\mathrm{Crc} / \mathrm{Crc}}$ or Celsr1 ${ }^{\mathrm{Crsh} / \mathrm{Crsh}}$ ) versus mutations in which there is complete loss of Vangl2 protein $\left(\right.$ Vangl $\left.^{L p / L p}\right)$ suggests that membrane-localized Vangl2 retains some ability to generate a polarizing signal, even in the absence of asymmetric localization, because the overall disruption in PCP is greater in Vangl2 $2^{L / L P}$ mutants.

\section{Interactions between Vangl2 and Scrb1 are required for asymmetric localization}

The results presented here also demonstrate a novel role for Scrb1 in the generation of protein asymmetry. Analysis of different deletion constructs indicates that the last three PDZ domains of Scrb1 directly interact with the C-terminal PDZ binding domain of Vangl2 and that this interaction is required for asymmetric localization of Vangl2. Interestingly, Vangl2 is still targeted to the membrane in Scrb $1^{\mathrm{Crc} / \mathrm{Crc}}$ mutants, although PDZ3 and PDZ4 are lost. However, because PDZ2 also interacts with Vangl2, it seems possible that $\mathrm{Scrb1}{ }^{\mathrm{Crc}}$ still retains some function.

Drosophila scrb restricts the localization of proteins to the apical cellular compartment during the generation of apicalbasal polarity, but the results presented here suggest that mammalian Scrb1 could also target or maintain proteins to a specific

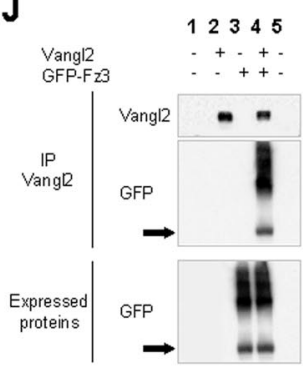

region of the membrane. Planar polarity and apical-basal polarity are probably concomitant processes, sharing molecules and molecular cascades. A recent paper showed the existence of an interaction between Drosophila PALS-1 associated tight junction protein, a protein from the apical marginal region junction, and $\mathrm{fz} 1$ in Drosophila eye, suggesting that molecules necessary for apical-basal polarity and planar polarity can interact (Djiane et al., 2005). Another recent paper analyzed the interactions of trilobite (tri)/stbm (a zebrafish allele of Vangl2) and scrb1 (Wada et al., 2005). The authors suggest that the two proteins could form a functional complex in which tri/stbm may be required for localization of scrb1 to the specific site of the membrane in which they are anchored and function. In our study, the disruption of Vangl2 asymmetry in Scrb1 $1^{\text {crc/crc }}$ mutants suggests that Scrb1 could be necessary to target or maintain Vangl2 to a specific region of the cellular membrane. The presence of four PDZ domains in the C-terminal tail of Scrb1 strongly suggests the existence of a large molecular complex, involving one or more additional proteins. These proteins could act in coordination with Scrb1 to control the asymmetric localization of Vangl2. In the future, it would be important to identify such molecules and their potential roles.

\section{Celsr1 is required for asymmetric localization of Vangl2}

The demonstration that Celsr 1 is required for the recruitment of Vangl2 to the membrane is consistent with the role of Flamingo in Drosophila. Flamingo has been shown to act at the top of the hierarchy of the PCP cascade and to be responsible for the membrane recruitment of other PCP proteins, including fz and stbm/ vang (Strutt, 2001; Bastock et al., 2003). In addition, a recent study demonstrated that chicken celsr 1 becomes asymmetrically localized to both proximal and distal edges of developing hair cells in the chicken basilar papilla, the functional equivalent of the cochlea (Davies et al., 2005). Finally, it is important to consider that we probably do not have a full understanding of the role of Celsr1 in the inner ear. The mammalian genome contains three separate Celsr genes, all of which are expressed in the cochlea (Shima et al., 2002). In addition, the Celsr $1^{\text {Crsh }}$ allele represents a point mutation in one of the extracellular cadherin repeats and therefore is probably hypomorphic rather than a complete loss of function. Consistent with this hypothesis, the overall disruption of PCP in cochleae from Celsr $1^{\text {Crsh/Crsh }}$ is not as extreme as in Vangl2 $^{L p / L p}$ (Curtin et al., 2003; Montcouquiol et al., 2003).

\section{Fz3 is asymmetrically localized and interacts with Vangl2}

The results presented here, along with the results presented by Wang et al. (2006), identify Fz3 and Fz6 as key regulators of PCP in the mammalian cochlea. As discussed, Fz3 and Fz6 share strong structural homology with Drosophila $\mathrm{fz}$, the $\mathrm{fz}$ that regulates PCP, and deletion of $F z 3$ and Fz6 leads to PCP defects in the inner ear (Wang et al., 2006). However, the location of Fz3, at the proximal edges of developing hair cells, and the dependence on 
A

Fly wing

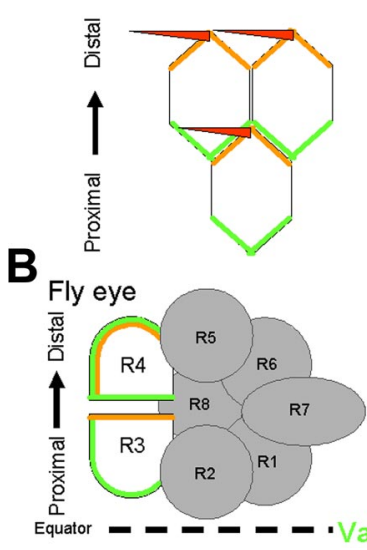

C

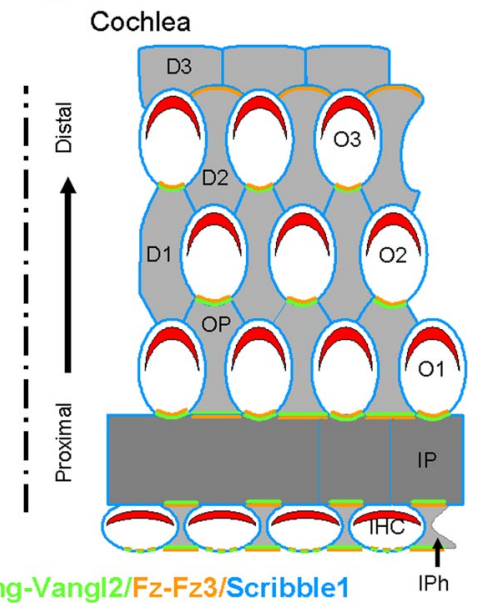

Figure 7. Asymmetric localization of Vangl2/vang, Fz3/fz, and Scribble1 in fly wing and eye and mammalian cochlea. $\boldsymbol{A}, \boldsymbol{B}$, Localization of $f z$ and vang in the Drosophila wing and eye. $A$, In the wild-type wing, a hair will form at the distal vertex on the apical surface of each cell and point distally. The actin-rich hair is believed to develop at the point of accumulation and activation of $f z$ (green) on the distal side. vang (orange) will localize on the opposite side (proximal). In the absence of $f z$ or vang, the asymmetry of the other protein is lost, and the hairs will form in the center of cells, pointing in the wrong direction. $\boldsymbol{B}$, In a wild-type eye, clusters of eight photoreceptors (R1-R8) called ommatidia become polarized, before they rotate according to the equator. Here again, a specific localization of $f z$ on the R3 side and of vang on the opposite R4 side is required for proper rotation, hence PCP. C, Lumenal view of a P0 mammalian organ of Corti. The stereociliary bundle (red) on each hair cell is located at the distal edge. Our data show that Vangl2 and Fz3 are localized opposite to the site of stereociliary bundle formation (the proximal side) of the IHCs and the $\mathrm{OHCs}(01-03)$. This accumulation of Vangl2 and Fz3 corresponds to the contact zone between a supporting cell and a hair cell and is especially strong for the outer hair cells. Our data suggest that, in fact, Vangl 2 and $\mathrm{Fz} 3$ are located on the membranes of both types of cells. The IPh cells, as well as the IPs and Ops, seems to express Vangl2 and Fz3 both proximal and distal, whereas the Deiters cells (D1-D3) seems to have only a distal expression. Scrb1 is expressed in every cell type.

Vangl2 for this asymmetry are markedly different from results obtained in Drosophila. In addition, the demonstration of a physical interaction between Vangl2 and Fz3 suggests that the loss of Fz3 asymmetry in the absence of Vangl2 is a result of a disruption in this physical interaction rather than a secondary effect of an overall disruption in cellular polarization. A previous paper showed that MAGI-3 (membrane-associated guanylate cyclase with inverted orientation-3) could function as a scaffold protein for Fz4 and Vangl2 to regulate the c-Jun N-terminal protein kinase cascade. However, whereas the authors identified a direct interaction between MAGI-3 and Fz4 or Fz7, no interaction was demonstrated between MAGI-3 and Fz3 or Fz6 nor between Fz4 and Vangl2 (Yao et al., 2004).

The observation that Fz3 (and possibly Fz6) colocalizes and interacts directly with Vangl2 suggests a fundamental change in the molecular mechanisms that regulate PCP between vertebrates and invertebrates. Moreover, this observation is at odds with the recent demonstration that $\mathrm{Dvl} 2$ becomes localized to the distal membranes of developing hair cells (Wang et al., 2005), unless other Fz receptors are present on the distal side of the hair cells. However the Dvl2 result was obtained using a transgenic animal rather than localization of the endogenous protein and so probably warrants additional study.

In summary, the results presented here demonstrate a key role for asymmetric localization of Vangl2 protein in the generation of PCP within the mammalian cochlea. We also uncovered new interactions between Fz3, Vangl2, and Scrb1 and the existence of

protein complexes. The targeting of these complexes to specific boundaries within the developing ear could generate a directional vector that would dictate the subsequent polarization of each cell within the structure. These data show that, although many aspects of PCP signaling have been conserved from invertebrates, significant changes have occurred at the cellular level in terms of how the polarizing signal is actually generated and how that signal then orchestrates actual polarization of a particular cell in mammals.

\section{References}

Adler PN (2002) Planar signaling and morphogenesis in Drosophilia. Dev Cell 2:525-535.

Adler PN, Lee H (2001) Frizzled signaling and cell-cell interactions in planar polarity. Curr Opin Cell Biol 13:635-640.

Bastock R, Strutt H, Strutt D (2003) Strabismus is asymmetrically localised and binds to Prickle and Dishevelled during Drosophila planar polarity patterning. Development 130:3007-3014.

Copp AJ, Checiu I, Henson JN (1994) Developmental basis of severe neural tube defects in the loop-tail $(L p)$ mutant mouse: use of microsatellite DNA markers to identify embryonic genotype. Dev Biol 165:20-29.

Curtin JA, Quint E, Tsipouri V, Arkell RM, Cattanach B, Copp AJ, Henderson DJ, Spurr N, Stanier P, Fisher EM, Nolan PM, Steel KP, Brown SD, Gray IC, Murdoch JN (2003) Mutation of Celsrl disrupts planar polarity of inner ear hair cells and causes severe neural tube defects in the mouse. Curr Biol 13:1129-1133.

Davies A, Formstone C, Mason I, Lewis J (2005) Planar polarity of hair cells in the chick inner ear is correlated with polarized distribution of c-flamingo-1 protein. Dev Dyn 233:998-1005.

Denman-Johnson K, Forge A (1999) Establishment of hair bundle polarity and orientation in the developing vestibular system of the mouse. J Neurocytol 28:821-835.

Djiane A, Yogev S, Mlodzik M (2005) The apical determinants aPKC and dPatj regulate Frizzled-dependent planar cell polarity in the Drosophila eye. Cell 12:621-631.

Fanto M, McNeill H (2004) Planar polarity from flies to vertebrates. J Cell Sci 117:527-533.

Hallworth R, McCoy M, Polan-Curtain J (2000) Tubulin expression in the developing and adult gerbil organ of Corti. Hear Res 139:31-41.

Hertzano R, Montcouquiol M, Rashi-Elkeles S, Elkon R, Yucel R, Frankel WN, Rechavi G, Moroy T, Friedman TB, Kelley MW, Avraham KB (2004) Transcription profiling of inner ears from Pou4f3(ddl/ddl) identifies Gfil as a target of the Pou4f3 deafness gene. Hum Mol Genet 13:2143-2153.

Kibar Z, Vogan KJ, Groulx N, Justice MJ, Underhill DA, Gros P (2001) Ltap, a mammalian homolog of Drosophila Strabismus/Van Gogh, is altered in the mouse neural tube mutant Loop-tail. Nat Genet 28:251-255.

Klein TJ, Mlodzik M (2005) Planar cell polarization: an emerging model points in the right direction. Annu Rev Cell Dev Biol 21:155-176.

Lu X, Borchers AG, Jolicoeur C, Rayburn H, Baker JC, Tessier-Lavigne M (2004) PTK7/CCK-4 is a novel regulator of planar cell polarity in vertebrates. Nature 430:93-98.

Montcouquiol M, Rachel RA, Lanford PJ, Copeland NG, Jenkins NA, Kelley MW (2003) Identification of Vangl2 and Scrb1 as planar polarity genes in mammals. Nature 423:173-177.

Murdoch JN, Doudney K, Paternotte C, Copp AJ, Stanier P (2001) Severe neural tube defects in the loop-tail mouse result from mutation of Lpp1, a novel gene involved in floor plate specification. Hum Mol Genet 15:2593-2601.

Navarro C, Nola S, Audebert S, Santoni MJ, Arsanto JP, Ginestier C, Marchetto S, Jacquemier J, Isnardon D, Le Bivic A, Birnbaum D, Borg JP (2005) Junctional recruitment of mammalian Scribble relies on E-cadherin engagement. Oncogene 24:4330-4339.

Qin Y, Capaldo C, Gumbiner BM, Macara IG (2005) The mammalian Scribble polarity protein regulates epithelial cell adhesion and migration through E-cadherin. J Cell Biol 171:1061-1071.

Rachel RA, Wellington SJ, Warburton D, Mason CA, Beermann F (2002) A new allele of Gli3 and a new mutation, circletail $(\mathrm{Crc})$, resulting from a single transgenic experiment. Genesis 33:55-61.

Sans N, Racca C, Petralia RS, Wang YX, McCallum J, Wenthold RJ (2001) Synapse-associated protein 97 selectively associates with a subset of 
AMPA receptors early in their biosynthetic pathway. J Neurosci 21:7506-7516.

Sans N, Prybylowski K, Petralia RS, Chang K, Wang YX, Racca C, Vicini S, Wenthold RJ (2003) NMDA receptor trafficking through an interaction between PDZ proteins and the exocyst complex. Nat Cell Biol 5:520-530.

Shima Y, Copeland NG, Gilbert DJ, Jenkins NA, Chisaka O, Takeichi M, Uemura T (2002) Differential expression of the seven-pass transmembrane cadherin genes Celsr $1-3$ and distribution of the Celsr2 protein during mouse development. Dev Dyn 223:321-332.

Simonneau L, Gallego M, Pujol R (2003) Comparative expression patterns of T-, N-, E-cadherins, beta-catenin, and polysialic acid neural cell adhesion molecule in rat cochlea during development: implications for the nature of Kolliker's organ. J Comp Neurol 459:113-126.

Strong LC, Hollander WF (1949) Hereditary loop-tail in the house mouse accompanied by inperforate vagina and with lethal craniorachischisis when homozygous. J Hered 40:329-334.

Strutt D (2003) Frizzled signalling and cell polarisation in Drosophila and vertebrates. Development 130:4501-4513.

Strutt DI (2001) Asymmetric localization of frizzled and the establishment of cell polarity in the Drosophila wing. Mol Cell 7:367-375.

Strutt DI (2002) The asymmetric subcellular localisation of components of the planar polarity pathway. Semin Cell Dev Biol 13:225-231.

Torban E, Wang H-J, Grouix N, Gros P (2004) Independent mutations in mouse Vang12 that cause neural tube defects in Looptail mice impair interactions with members of the disheveled family. J Biol Chem 279: 52703-52713.

Uemura T, Shimada Y (2003) Breaking cellular symmetry along planar axes in Drosophila and vertebrates. J Biochem (Tokyo) 134:625-630.

Wada H, Iwasaki M, Sato T, Masai I, Nishiwaki Y, Tanaka H, Sato A, Nojima Y, Okamoto H (2005) Dual roles of zygotic and maternal Scribble1 in neural migration and convergent extension movements in zebrafish embryos. Development 132:2273-2785.

Wang J, Mark S, Zhang X, Qian D, Yoo SJ, Radde-Gallwitz K, Zhang Y, Lin X, Collazo A, Wynshaw-Boris A, Chen P (2005) Regulation of polarized extension and planar cell polarity in the cochlea by the vertebrate PCP pathway. Nat Genet 37:980-985.

Wang Y, Guo N, Nathans J (2006) The role of Frizzled3 and Frizzled6 in neural tube closure and in the planar polarity of inner-ear sensory hair cells. J Neurosci 26:2147-2156.

Woods C, Montcouquiol M, Kelley MW (2004) Mathl regulates development of the sensory epithelium in the mammalian cochlea. Nat Neurosci 7:1310-1318.

Yao R, Natsume Y, Noda T (2004) MAGI-3 is involved in the regulation of the JNK signaling pathway as a scaffold protein for frizzled and Ltap. Oncogene 23:6023-6030. 